\title{
Assessing the degree of plug flow in oxidation flow reactors (OFRs): a study on a Potential Aerosol Mass (PAM) reactor
}

Dhruv Mitroo ${ }^{1, a, *}$, Yujian Sun ${ }^{1, *}$, Daniel P. Combest ${ }^{2}$, Purushottam Kumar ${ }^{3}$, and Brent J. Williams $^{1}$

${ }^{1}$ Department of Energy, Environmental \& Chemical Engineering, Washington University in St. Louis, St. Louis, MO, USA

${ }^{2}$ ENGYS, Ltd.

${ }^{3}$ Department of Chemical Engineering, Indian Institute of Technology Gandhinagar, Palaj, Gujarat 382355, India

${ }^{a}$ Now at the Department of Atmospheric Sciences, Rosenstiel School of Marine and Atmospheric Sciences, University of Miami, Miami, FL, USA

*equally contributing authors

Correspondence to: brentw@wustl.edu

Brent J. Williams, Ph.D.

Raymond R. Tucker I-CARES Career Development Associate Professor

Department of Energy, Environmental \& Chemical Engineering

Washington University in St. Louis 


\section{Supplemental information}

\section{S1. Data collection}

Accurate RTDs depend on proper methodology for tracer tests. Obtaining abundant data is preferable to obtaining scarce data. We therefore obtained the first and second moments of the ECurve by:

$$
\begin{gathered}
\bar{t}=\frac{\sum_{i} t_{i} C_{i} \Delta t_{i}}{\sum_{i} C_{i} \Delta t_{i}}, \\
\sigma^{2}=\frac{\sum_{i} t_{i}^{2} C_{i} \Delta t_{i}}{\sum_{i} C_{i} \Delta t_{i}}-\bar{t}^{2},
\end{gathered}
$$

which are the discrete forms of Eqs. (A10) and (A12). Scarce data would have required different treatment and a choice to use instantaneous data or mixing cup readings (Levenspiel, 2002).

The 'step-up' experiment, described in the first paragraph of Sect. 3.1, required only one action: opening the $\mathrm{SO}_{2}$ MFC to a desired value at an arbitrary time $t_{0}$. Theoretically, obtaining an ECurve from F-Curve data is as simple as Eq. (A2), however numerically we applied Simpson's rule for a discrete dataset. Due to instabilities in the $\mathrm{SO}_{2}$ detector, if we applied this to a direct 'step-up', the tail of the E-Curve would be very noisy. So we did a step-down (i.e., we closed the $\mathrm{SO}_{2}$ tracer MFC at time $t_{0}$ after its signal reached steady state), and then flipped the curve (now representative of a step-up) (Levenspiel, 2002), and then took its derivative. This way, the magnitude in fluctuation at the tail end of the E-Curve was suppressed, and the E-Curve became smooth. 
S2 snappyHexMesh mesh

\begin{tabular}{|c|c|}
\hline Total Number of Cells & 917100 \\
\hline Hexahedral & 898092 \\
\hline Prisms & 18160 \\
\hline Polyhedral & 848 \\
\hline Typical Cell Dimensions (mm) & 2.5 \\
\hline
\end{tabular}

Table S1: Dimensions for the mesh used in CFD analysis.

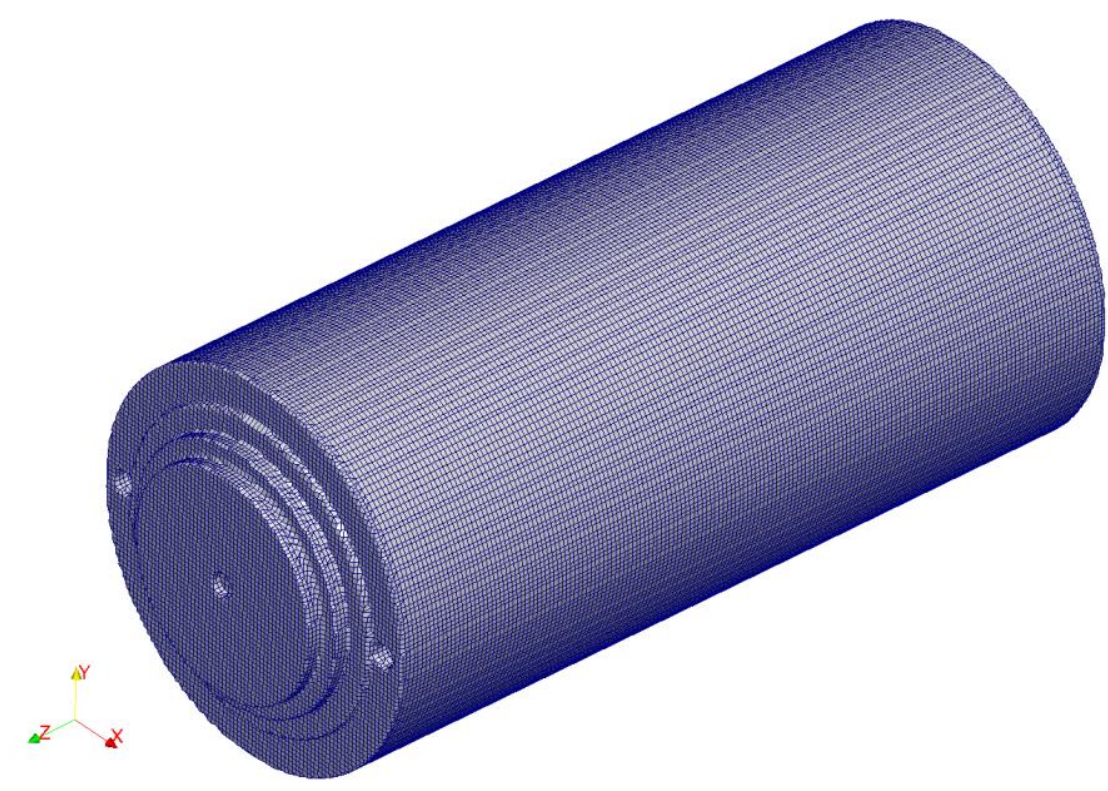

Figure S1: Mesh for the PAM.

\section{S3 TIS Model}




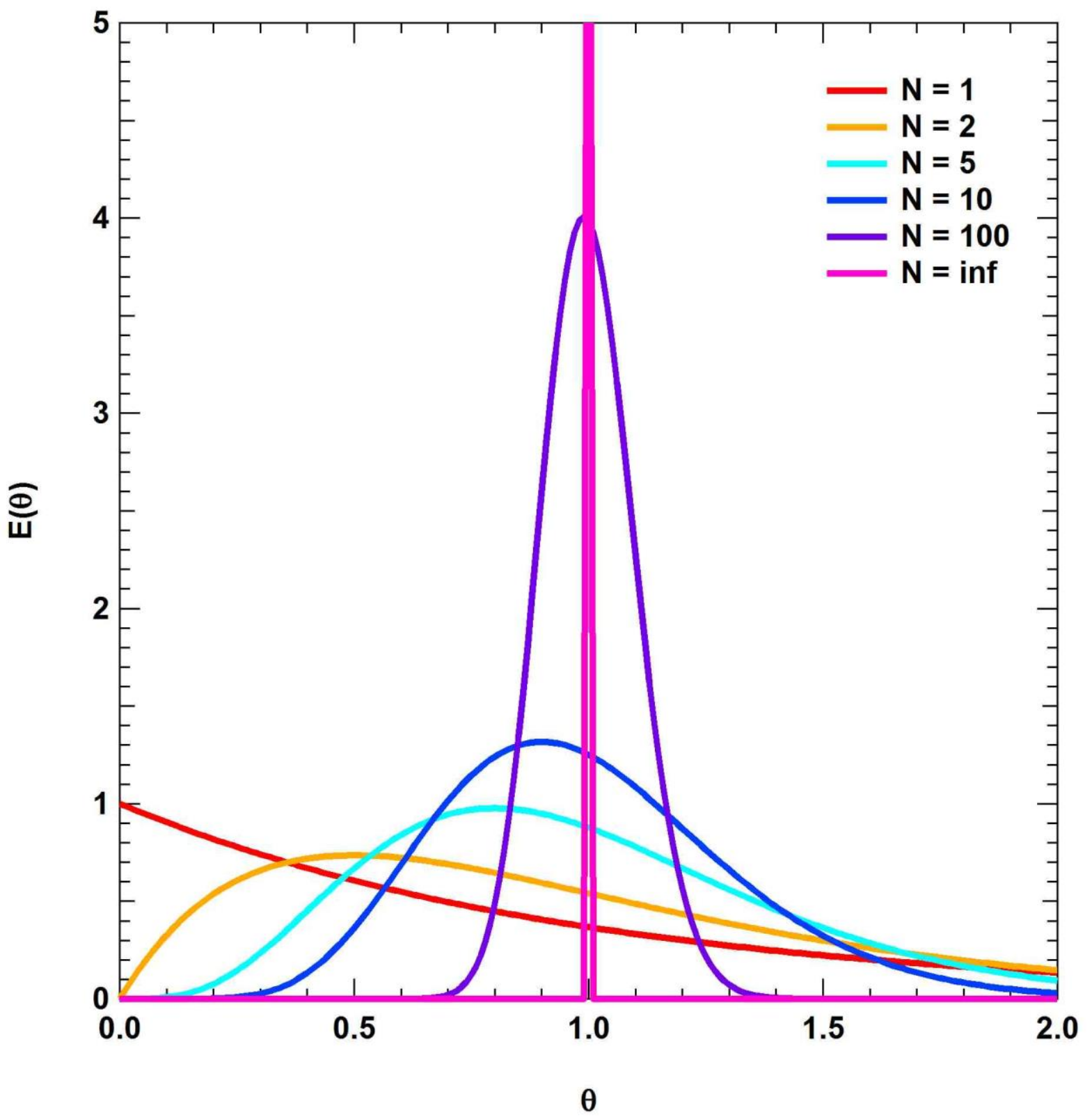

Figure S2: RTD shape as a function of $N$ for the TIS model. 


\section{S4 Dimensionless numbers}

If the PAM can be engineered so the ADM describes it well, some dimensional analysis that can help optimize its use. First, to see if the ADM is satisfied by a cylinder the size of the PAM, we can use the relationship that compares its characteristic advective transport rate to its characteristic diffusive transport rate. We can obtain this by matching value of the Taylor diffusivity to the molecular diffusivity (Aris, 1956; Taylor, 1954):

$$
\frac{\bar{u}^{2} R^{2}}{48 \mathcal{D}}>\mathcal{D}
$$

where $\bar{u}$ is the mean cross-sectional velocity, $R$ is the PAM's radius, and $\mathcal{D}$ is the molecular diffusivity of the tracer, $\mathrm{SO}_{2}$, and the collective term on the left hand side (LHS) is the Taylor diffusion coefficient. Equation (S3) can be rearranged to yield

$$
\frac{\bar{u} R}{\mathcal{D}}>\sqrt{48}
$$

where the LHS of Eq. (S4) is the radial Péclet number (Pér). Both the Washington University PAM and the Pennsylvania State University PAM are eight inches in inner diameter (ID). We use a value of $\mathcal{D}$ of $0.146 \mathrm{~cm}^{2} \mathrm{~s}^{-1}$ (Yaws, 2010) for $\mathrm{SO}_{2}$ in air at $298 \mathrm{~K}$ to mimic the diffusivity of the tracer in the carrier gas in the PAM. Equation (S4) holds true for a flowrate greater than 2 LPM given this geometry, so we validate the use of ADM for a cylinder the size of the Washington Unviersity PAM as long as it is operated above 2 LPM.

The upper limit is dictated by the aspect ratio, where 


$$
\frac{4 L}{A}>P e_{r}^{\prime},
$$

where $A$ is the cross sectional area of the PAM. Equation (S5) remains valid for just under 16 LPM.

\section{References}

Aris, R.: On the Dispersion of a Solute in a Fluid Flowing through a Tube, Proc. R. Soc. Math. Phys. Eng. Sci., 235(1200), 67-77, doi:10.1098/rspa.1956.0065, 1956.

Levenspiel, O.: The chemical reactor omnibook, OSU Book Stores, Corvallis, Or., 2002.

Taylor, G. I.: Diffusion and Mass Transport in Tubes, Proc. Phys. Soc. Sect. B, 67(12), 857-869, doi:10.1088/0370-1301/67/12/301, 1954.

Yaws, C. L.: Yaws' transport properties of chemicals and hydrocarbons, Knovel, Norwich, N.Y. [online] Available from: http://app.knovel.com/hotlink/toc/id:kpYTPCHEE8/yaws-transportproperties (Accessed 18 December 2015), 2010. 